\title{
La variazione seconda generalizzata nel caso dei punti terminali mobili e problemi sugli autovalori connessi.
}

\author{
Memoria di Gabriele Mammana (a Napoli).
}

Sunte. - L'A. prendendo in esame un funzionale J[y] l' integrale di una espressione quadratica in $\mathrm{y}$ e $\left.\mathrm{y}^{\prime}\right)$, per $\mathrm{y}$ variabile nell'insieme delle funzioni di classe $\mathrm{C}^{\prime}$ in un dato inter: vallo, mette in rilievo taluni problemi sugli autovalori inerenti a sistemi differenziali con condizioni quadratiche in due punti o generali condizioni lineari agli estremi, ed assegna, seguendo un procedimento assai semplice, una condizione espressiva e di facile applicazione che è necessaria e sufficiente: per l'esistenza del minimo di J[y] e di autovalori contenuti nell' intervallo $(0,1)$.

Nel contempo dimostra l'esistenza di ?nfiniti autovalori (con l'infinito come punto limite) pei menzionati sistemi, e, richiamandosi a precedenti studi, rileva la possibilitò di estendere le superiori considerazioni al caso che si debba decidere dell' esistenza o no di autovalori contenuti in un intervallo assegnato $\left(\mathrm{r}_{1}, \mathrm{r}_{2}\right)$ e che il parametro figuri non linearmente.

\section{Introduzione.}

In un lavoro del 1919 L. Lichtenstein ( $\left.{ }^{1}\right)$ si pone fra l'altro il problema di minimizzare l'integrale

$$
\begin{gathered}
J[y]=\int_{x_{1}}^{x_{2}}\left(a y^{\prime 2}+2 b y y^{\prime}+c y^{2}+p y^{\prime}+q y+\partial\right) d x, \\
\text { per } \quad p=q=\partial=0, \quad a>0, \quad \text { in }\left(x_{1}, x_{2}\right)
\end{gathered}
$$

nell' insieme, $(G)$, di tutte le curve di classe $C^{\prime}$ (a punti terminali mobili) aventi l'intervallo $\left(x_{1}, x_{2}\right)$ come base.

Successivamente ebbero ad occuparsi dello stesso problema, nell'ipotesi (più generale) che l'integrando di $J[y]$ fosse non una forma ma una funzione quadratica la più generale in $y$ e $y^{\prime}$, MaUro Picone, per incidenza, $\left(^{2}\right)$ e il suo allievo E. De Agostini ( $\left.{ }^{3}\right)$.

(') L. Lichtenstein, Zur variationrechnung. "Nachrichten von dor Koniglichen Gesell. schaft der Wissenschaften zu Gottingen », 1919, p. 47.

(2) M. Picone, Lezioni di calcolo delle variazioni, "Circolo Matematico di Catania», 1924, pp. 47.

(3) E. $\mathrm{D}_{\mathrm{e}}$ Agostino, Particolare problema di calcolo delle variazioni, "R. Istituto Lom. bardo etc. », V. LXV, fasc. VI-X, 1932, pp. 405-423. 
Lichtensteis, facendo ricorso a teoremi di Schmidt sugli sviluppi in serie di funzioni ortogonali, perviene, con metodo piuttosto complicato e artifizioso, alla conclusione seguente.

Affinchè $J[y]$ abbia minimo in $(G)$ oceorre e basta che:

a) il più piccolo autovalore positivo, $\rho$, dell' equazione (associata a quella di JACOBI)

$$
\left(a u^{\prime}\right)^{\prime}+\rho\left(b^{\prime}-c\right) u=0
$$

risulti maggiore o uguale a uno,

b) risulti pure maggiore o uguale ad uno il più piccolo valore (eccezionale) positivo, $\rho$, per il quale è possibile soddisfare, con soluzioni diverse da quella nulla identicamente, al sistema differenziale (con due distinte condizioni lineari agli estremi)

$$
\left\{\begin{array}{l}
\left(a u^{\prime}\right)^{\prime}+Q \rho\left(b^{\prime}-c-Q\right) u=0 \\
\left(a u^{\prime}+b u\right)_{x_{1}}=\left(a u^{\prime}+b u\right)_{x_{2}}=0
\end{array}\right.
$$

(con $Q$ avendo designato una funzione essenzialmente negativa e del resto qualsiasi).

Nei riguardi del criterio contenuto in questa conclnsione è da rilevare innanzi tutto:

la condizione $a$ ) è difettosa, chè, come è provato in principio del presente lavoro, il funzionale $J[y]$ non ha mai minimo nel caso che il più piccolo autovalore positivo della (I) sia uno.

A parte questo, osserviamo che, mentre è possibile dall' andamento di un integrale particolare dell' equazione (di JAcoBr)

$$
\left(a u^{\prime}\right)^{r}+\left(b^{\prime}-c\right) u=0
$$

assicurare o escludere l'esistenza di autovalori per la (I) contenuti nell' intervallo $(0,1)$, non si ha alcun mezzo del genere atto ad assicnrare l'esistenza o la non esistenza di valori eccezionali del sistema (II) che cadono nel menzionato intervallo. Sicchè la verifica della condizione b) presenta in generale difficoltà insormontabili.

Del resto a giudicare dell' efficacia del criterio basta il fatto ehe per esso, ad esempio, non è possibile decidere, in un caso banale come quello in cui l'equazione di EuLERo si ridu’ee alla sua forma più semplice:

$$
\left(a u^{\prime}\right)^{\prime}=0 \text {, }
$$

se esiste e sotto quali condizioni esiste minimo per $J[y]$.

Il criterio quindi avrebbe carattere puramente teorico, e la sua utilità, in un problema delle applicazioni, è pertanto discutibile. 
Come ho accennato sopra, si occupò di questo problema, nell' ipotesi più generale menzionata, anche M. PICONE, il quale, con procedimento quanto mai semplice ed elegante, trattò il caso dei punti terminali fissi conseguendo i noti definitivi risultati.

Successivamente E. DE Agostrno tentò di applicare i procedimenti introdotti con successo in questa e altre teorie da Picone per studiare il problema di estremare $J[y]$ (nell'ipotesi più generale considerata dal PICONE stesso) nel caso dei punti terminali mobili.

Ma quest' autore non conseguì risultati apprezzabili

Un criterio di sufficienza (l'unico) raggiunto dal DE Agosmixo contiene una condizione [esistenza di un integrale del sistema formato dalla (I) e dalle condizioni (II), che si mantiene diverso da zero in tutto l'intervallo $\left.\left(x_{1}, x_{2}\right)\right]$ la quale oltre ad essere di natura teorica è probabilmente irrealizzabile ( $\left.{ }^{1}\right)$.

Del resto, la poca attendibilità del risultato può desumersi dal fatto che esso, nel caso banale sopra menzionato a proposito del eriterio di LIOHTENSTEIN, non da modo di raggiungere la conclusione.

Ho creduto quindi opportuno un rigoroso esame della questione allo scopo di cercare condizioni, non teoriche, semplici - di verifica possibile atte ad assicurare o ad escludere l'esistenza di minimo del funzionale $J[y]$ (nell'ipotesi che l'integrando sia una funzione quadratica in $y$ e $y^{\prime}$ la più generale e $y(x)$ vari nell' insieme $(G)$ di tutte le curve di classe $C^{\prime}$, a punti terminali mobili, aventi $\left(x_{1}\right.$ e $\left.x_{2}\right)$ per base $)$ e nello stesso tempo dedurre teoremi di esistenza di autovalori, contenuti in un dato intervallo, relativi a sistemi formati da una equazione del secondo ordine, dipendente da un parametro, con una condizione quadratica in due punti, o due condizioni lineari (le più generali) distinte agli estremi. Teoremi intimamente collegati alla questione menzionata.

Il risultato finale conseguito nei riguardi del funzionale $J$ è importante anche perchè assegna una condizione necessaria, in forma quanto mai semplice, per l'esistenza di minimo in un problema qualsiasi di calcolo delle variazioni nel quale i punti terminali della curva variabile siano mobili su due rette normali all' asse delle $x$. I principali risultati conseguiti nel presente lavoro sono espressi dai seguenti teoremi

Teonema $1^{\circ}$ - Condizione necessaria e sufficiente perchè l'integrale $J$ abbia minimo in $(G)$ è che

a) l'intervallo $\left(x_{1}, x_{2}\right)$, estremi inclusi, sia privo di punti coniugati rispetto all' equazione (di J ACOBI) $\left(a u^{\prime}\right)^{\prime}+\left(b^{\prime}-c\right) u=0$.

(1) $L$ 'autere non si preoccupa di assicurarsi se tale condizione è effettivamente realizzabile. 
b) detti $u, v$ due integrali indipendenti di questa equazione risulti

$$
\left|\begin{array}{cc}
u_{2} & v_{2} \\
-u_{1} & -v_{1}
\end{array}\right| \begin{array}{ll}
a_{2} u_{2}{ }^{\prime}+b_{2} u_{2} & a_{2} v_{2}{ }^{\prime}+b_{2} v_{2} \\
a_{1} u_{1}{ }^{\prime}+b_{1} u_{1} & a_{1} v_{1}{ }^{\prime}+b_{1} v_{1}
\end{array} \mid>0 \quad\left[u\left(a u^{\prime}+b u\right)\right]_{x_{1}}^{x_{2}}>0,\left(^{1}\right) .
$$

c) oppure insieme ad a) sia soddisfatta quest'altra

$$
\left\|\begin{array}{lll}
a_{2} u_{2}^{\prime}+b_{2} u_{2} & a_{1} u_{1}^{\prime}+b_{1} u_{1} & {[p u]_{x_{1}}^{x_{2}}-\int_{x_{1}}^{x_{2}}\left(p^{\prime}-q\right) u d x} \\
a_{2} v_{2}^{\prime}+b_{2} v_{2} & a_{1} v_{1}^{\prime}+b_{1} v_{1} & {[p v]_{x_{1}}^{x_{\bar{\sigma}}}-\int_{x_{1}}^{x_{2}}\left(p^{\prime}-q\right) u d x}
\end{array}\right\|^{2} \quad\left[\begin{array}{cc}
{\left[u\left(a u^{\prime}+b u\right)\right]_{x_{1}}^{x_{2}}>0} & {\left[v\left(a v^{\prime}+b v\right)\right]_{x_{1}}^{x_{2}}>0 .}
\end{array}\right.
$$

Nel caso a), b) esiste una e una sola curva minimante $J$ in $(G)$.

Nel caso $a$ ), c) esiste una semplice infinità di curve minimanti.

Teorema $2^{\circ}$ - Il realizzarsi delle condizioni $a$ ) e b), o $a$ ) e c) è condizione necessaria e sufficiente perchè resti esclusa, pel sistema differenziale

$$
\begin{cases}\left(a u^{\prime}\right)^{\prime}+\rho(b-c) u=0 & 0<\rho \leq 1 \\ {\left[u\left(a u^{\prime}+\rho b u\right)\right]_{x_{1}}^{x_{2}} \leq 0,} & \end{cases}
$$

e per l'altro analogo con due distinte condizioni lineari agli estremi, l'esistenza di soluzioni diverse da quella identicamente nulla.

Si trae poi occasione dall' esame della presente questione di calcolo delle variazioni per dimostrare (parte II), in modo molto semplice, l'esistenza di infiniti valori eccezionali, aventi l'infinito come punto limite, pei quali è assicurata l'esistenza di soluzioni, non nulle, pei sistemi del tipo seguente

$$
\left\{\begin{array}{l}
\left(a u^{\prime}\right)^{\prime}+p k u=0 \\
{\left[u\left(a u^{\prime}+p b u\right)\right]_{x_{2}}^{x_{u}} \lesssim 0}
\end{array}\right.
$$

o di quest'altro

$$
\left\{\begin{array}{l}
\left(a u^{\prime}\right)^{\prime}+\rho k u=0 \\
\left(a u^{\prime}+\rho b u\right)_{x_{1}}=\left(a u^{\prime}+\rho b u\right)_{x_{2}}=0
\end{array}\right.
$$

etc, etc...., con $a$ e $b$ qualsiasi ete.

Notiamo infine la possibilịtà, che si deduce dalle considerazioni fatte nella presente memoria, di estendere $i$ teoremi sugli autovalori al caso che l'intervallo dove si vuole sia contenuto $\rho$ sia qualsiasi, e sia pure qualsíasi, e non soltanto lineare, la dipendenza del coefficiente di $u$ dal parametro $\rho$.

(t) Con $a_{1}, b_{1}, u_{1}, v_{1}$, etc., e $a_{2}, b_{2}, u_{2}, v_{2}$, etc., sono designati i valori di $a, b, u, v$, ete., rispettivamente in $x_{1}$ e $x_{2}$. 
1. Relativamente al funzionale

$$
J[y]=\int_{x_{1}}^{x_{2}}\left(a y^{\prime 2}+2 b y y^{\prime}+c y^{2}+2 p y^{\prime}+2 q y+\jmath\right) d x,
$$

[per $y$ variabile nell'insieme, $(G)$, delle funzioni di classe $C^{\prime}$ in $\left(x_{1}, x_{2}\right)$ ] supporremo

$$
a, b, c, p, q, \partial
$$

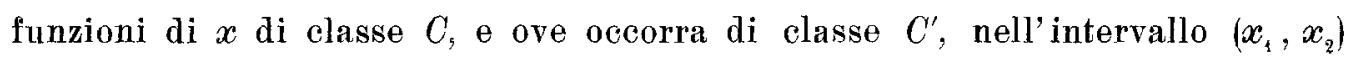
ed $a$ di segno costante; per fissare le idee

$$
a(x)>0 \quad \text { per } \quad x_{1} \leq x \leq x_{2} \quad(1) .
$$

Poichè un' eventuale estremante di $J[y]$ in $(G)$ è indipendente da $\partial$, possiamo senz'altro supporre questo coefficiente identicamente nullo.

Un qualunque elemento di $(G)$ si può sempre pensare rappresentato mediante una somma del tipo

$$
y=g(x)+\varepsilon z(x),
$$

con $g$ e $z d$ i classe $C^{\prime}$ in $\left(x_{1}, x_{2}\right)$ ed $\varepsilon$ costante. Si ha allora:

$$
\begin{gathered}
J[g+\varepsilon z]=J[g]+\varepsilon_{\dot{x}_{1}^{2}}^{x_{2}}\left(a z^{\prime 2}+2 b z z^{\prime}+c z^{2}\right) d x+2 \varepsilon \int_{x_{1}}^{x_{2}}\left(a g^{\prime} z^{\prime}+b(g z)^{\prime}+c g z+p z^{\prime}+q z\right) d x= \\
=J[g]+\varepsilon^{2} \cdot \grave{x}_{x_{1}}^{x_{2}}\left(\alpha z^{\prime 2}-\left\{b^{\prime}-c\right\} z^{2}\right) d x+2 \varepsilon \int_{x_{1}}^{x^{3}}\left(a g^{\prime} z^{\prime}-\left\{b^{\prime}-c\right\} g z-\left\{p^{\prime}-q\right\} z\right) d x+\varepsilon^{2}\left[b z^{2}\right]_{x^{2}}^{x_{2}} \\
+2 \varepsilon[(b g+p)]_{x_{1}}^{x_{2}} .
\end{gathered}
$$

Assumiamo come funzione $z$ un integrale dell' equazione (dipendente da un parametro $p$ )

$(4)_{f}$

$$
\left(a z^{\prime}\right)^{\prime}+f \cdot\left(b^{\prime}-c\right) z=0 .
$$

Per $p \neq 0$, e posto dunque che $z$ soddisfi a questa equazione, dalle identità

$$
\frac{1}{\rho}\left(a z^{\prime}\right)^{\prime} z=-\left(b^{\prime}-c\right) z^{2}, \quad \frac{1}{\rho}\left(a z^{\prime}\right)^{\prime} g=-\left(b^{\prime}-c\right) z g,
$$

(1) Il problema posto ¿̀ quindi regolare. 
integrando fra $x_{1}$ e $x_{2}$, ricaviamo

$$
\begin{aligned}
& \frac{1}{\rho}\left[a z z^{\prime}\right]_{x_{1}}^{x_{2}}-\frac{1}{\rho} \int_{x_{1}}^{x_{2}^{\prime}} a z^{\prime 2} d x=-\int_{x_{1}}^{x_{2}}\left(b^{\prime}-c\right) z^{\prime} d x \\
& \frac{1}{\rho}\left[\left.a g z^{\prime}\right|_{x_{1}} ^{x_{2}}-\frac{1}{\rho} \int_{x_{1}}^{x_{2}} a z^{\prime} g^{\prime} d x=-\int_{x_{1}}^{x_{2}}\left(b^{\prime}-c\right) z g d x,\right.
\end{aligned}
$$

da cui, sostituendo della (2),

$$
\begin{gathered}
J[g+\varepsilon z]=J[g]+\varepsilon^{2}\left(1-\frac{1}{\rho}\right) \int_{x_{1}}^{x_{3}} a z^{\prime 2} d x+\frac{\varepsilon^{2}}{\rho}\left[z\left(a z^{\prime}+\rho b z\right)\right]_{x_{1}}^{x_{2}}+ \\
+2 \varepsilon\left(1-\frac{1}{\rho}\right) \int_{x_{1}}^{x_{2}} a z^{\prime} g^{\prime} d x+2 \varepsilon\left\{\frac{1}{\rho}\left|g\left(a z^{\prime}+\rho b z\right)\right|_{x_{1}}^{x_{2}}+\left[p z \int_{x_{1}}^{x_{2}}-\int_{x_{1}^{\prime}}^{x_{2}}\left(p^{\prime}-q\right) z d x\right\} .\right.
\end{gathered}
$$

Da questa relazione deduciamo

$\left.1^{\circ}\right)$ Il funzionale $\mathrm{J}[\mathrm{y}]$ non ha massimo in (G), chè esistendo, come verificheremo più avanti, infiniti valori (eccezionali) reali di $\rho$, di modulo maggiore di uno, pei quali è possibile soddisfare al sistema differenziale

$$
\begin{aligned}
& \left(a z^{\prime}\right)^{\prime}+\rho\left(b^{\prime}-c\right) z=0 \\
& {\left[z\left(a z^{\prime}+\rho b z\right)\right]_{x_{1}}^{x_{2}}=0}
\end{aligned}
$$

$$
|P|>1
$$

con soluzioni, $\bar{z}$, diverse da quella identicamente nulla, dalla (3), per ognuna di queste soluzioni, si trae (a causa della (1) e comnnque sia fissata $g$ )

ciò che prova l'asserto

$$
\lim _{\varepsilon \rightarrow \infty} J[g+\bar{\varepsilon} \bar{z}]=+\infty,
$$

2o) Il funzionate J[y] è sfornito anche di minimo in (G) nell'ipotesi che esista una soluzione, diversa da quella nulla, del sistema differenziale

(6)

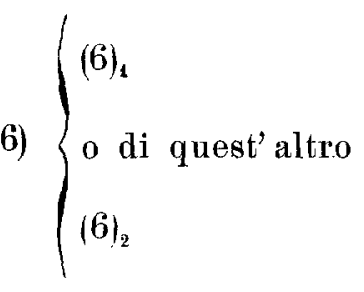

$$
\begin{aligned}
& \left\{\begin{array}{ll}
\left(a z^{\prime}\right)^{\prime}+\rho\left(b^{\prime}-c\right) z=0 & \text { con } 0<\rho<1 \\
{\left[z\left(a z^{\prime}+\rho b z\right)\right]_{x_{1}}^{x_{2}} \leq 0} & \text { con } \rho=1
\end{array}\right\} \\
& \left\{\begin{array}{l}
\left(a z^{\prime}\right)^{\prime}+\rho\left(b^{\prime}-c\right) z=0 \\
{\left[z\left(a z^{\prime}+\rho b z\right)\right]_{x_{1}}^{x_{2}}<0}
\end{array}\right.
\end{aligned}
$$

$\left.3^{\circ}\right)$ Ove esista, infine, una soluzione, non nulla identicamente, del sistema

$$
\left\{\begin{array}{l}
\left(a z^{\prime}\right)^{\prime}+\left(b^{\prime}-c\right) z=0 \\
{\left[z\left(a z^{\prime}+b z\right)\right]_{x_{1}}^{x_{3}}=0}
\end{array}\right.
$$


l'esistenza del minimo per $\mathrm{J}$ è pure da escludere in generale, salvo eventualmente nel caso in cui per ogni soluzione, non nulla, $z$, di questo sistema (7) si abbia simultaneamente

$$
\left(a z^{\prime}+b z\right)_{x_{1}}=\left(a z^{\prime}+b z\right)_{x_{2}}=\left[p z \int_{x_{1}}^{x_{2}}-\int_{x_{1}^{\prime}}^{x_{2}}\left(p^{\prime}-q\right) z d x=0\right.
$$

quindi, in particolare, il sistema (7) abbia come conseguenza quest'altro (con condizioni distinte agli estremi)

$$
\left\{\begin{array}{l}
\left(a z^{\prime}\right)^{\prime}+\left(b^{\prime}-c\right) z=0 \\
\left(a z^{\prime}+b z\right)_{x_{1}}=\left(a z^{\prime}+b z\right)_{x_{2}}=0 .
\end{array}\right.
$$

Invero, nell'ipotesi che si possa soddisfare a (7) con una funzione $z$ non nulla identicamente, la (3) diventa

(9) $J[g+\varepsilon z]=J[g]+2 \varepsilon\left\{g\left(x_{2}\right)\left(a z^{\prime}+b z\right)_{x_{2}}-g\left(x_{1}\right)\left(a z^{\prime}+b z\right)_{x_{1}}+[p z]_{x_{1}}^{x_{2}}-\int_{x_{2}}^{x_{2}}\left(p^{\prime}-q\right) z d x\right\}$.

Il secondo membro di questa relazione, nel caso che per $z$ non si suppongano soddisfatte simultaneamente le (8), è lineare, nei valori, $g_{1}$ ed $g_{2}$, di $g(x)$ agli estremi, valori che possiamo supporre, per l'arbitrarietà di $g$, tali da rendere il coefficiente di $2 \varepsilon$, nella (9), diverso da 0 .

Dalla (9) stessa si trae allora, facendo variare $\varepsilon$ da $-\infty$ a $+\infty$,

$$
\begin{aligned}
& \text { estremo inf. } J(g+\varepsilon z)=-\infty \\
& \text { estremo sup. } J(g+\varepsilon z)=+\infty
\end{aligned}
$$

c. v. d.

4) Il superiore ragionamento cade in difetto, com'è chiaro, nel caso segnalato; cioè che il sistema (7) abbia come conseguenza le (8).

Raggruppiamo i precedenti risultati nel seguente

Teorema I. - a) Il funzionale $J[y]$ non ha massimo in $(G)$.

b) Perchè il medesimo funzionale $J$ abbia minimo in $(G)$ occorre che non esistano ad eccezione della soluzione nulla altre soluzioni sia del sistema differenziale

$(6)_{1}$

$$
\left\{\begin{array}{l}
\left(a z^{\prime}\right)^{\prime}+f\left(b^{\prime}-c\right) z=0 \\
{\left[z\left(a z^{\prime}+p b z\right)\right]_{x_{1}}^{x_{2}} \leq 0}
\end{array}\right.
$$

$$
\operatorname{con} 0<\rho<1
$$

sia di quest'altro

$(6)_{2}$

$$
\left\{\begin{array}{l}
\left(\alpha z^{\prime}\right)^{\prime}+\rho\left(b^{\prime}-c\right) z=0 \\
{\left[z\left(a z^{\prime}+\rho b z\right)\right]_{x_{1}}^{x_{2}}<0,}
\end{array}\right.
$$$$
\operatorname{con} \rho=1
$$ 
c) Nell'ipotesi poi che esistano soluzioni, diverse da quella nulla, del sistema

$$
\begin{array}{ll}
\left(a z^{\prime}\right)^{\prime}+p\left(b^{\prime}-c\right) z=0 & \text { con } \rho=1 \\
{\left[z\left(a z^{\prime}+p b z\right)\right]_{x_{1}}^{x_{2}}=0,} &
\end{array}
$$

condizione necessaria perchè $J$ sia fornito di minimo in $(G)$, è che ciascuna di queste soluzioni sia anche soluzione di quest'altro sistema differenziale, con tre condizioni distinte agli estremi,

$$
\begin{gathered}
\left(a z^{\prime}\right)^{\prime}+\left(b^{\prime}-c\right) z=0 \\
\left(a z^{\prime}+b z\right)_{x_{2}}=\left(a z^{\prime}+b z\right)_{x_{1}}=[p z]_{x_{1}}^{x_{2}}-\int_{x_{1}}^{x_{2}}\left(p^{\prime}-q\right) d x=0
\end{gathered}
$$

Supponiamo in particolare $x_{1}$ e $x_{2}$ coniugati rispetto all' equazione

$$
\left.\left(a z^{\prime}\right)^{\prime}+\left(b^{\prime}-c\right) z=0,1^{1}\right)
$$

allora ogni soluzione di quest ultima nulla agli estremi soddisfa al sistema (7) ma non al sistema (8); chè

$$
\text { se } \left.\quad z\left(x_{1}\right)=z\left(x_{2}\right)=0, \quad z^{\prime}\left(x_{j}\right) \neq 0 \quad \text { (per } \quad j=1,2\right) .
$$

Ne consegue che

se $\mathrm{x}_{1}$ e $\mathrm{x}_{2}$ sono coniugati rispetto all' equazione (di Jacobi) (4), l'integrate $\mathrm{J}[\mathrm{y}]$ non ha minimo in (G).

Com'è noto, un eventuale valore di $\rho$ per cui esista un integrale dell'equazione

$$
\left(a z^{\prime}\right)^{\prime}+\rho\left(b^{\prime}-c\right) z=0
$$

nullo in $x_{1}$ e $x_{2}$ e diverso da quello identicamente nullo, dicesi un autovalore rispetto a questa equazione, e l'integrale corrispondente (nullo agli estremi detti) (') un' autosoluzione.

Il precedente teorema ha pertanto, in particolare, come conseguenza il seguente

Corollario (prima furma).

Condizione necessaria perchà $J[y]$ sia dotato di minimo in $(G)$ è che il più piccolo antovalore positivo della $(4)_{\rho}$ sia maggiore di uno.

Ma perchè ciò abbia luogo occorre e basta che l'intervallo $\left(x_{1}, x_{2}\right)$, estremi

(1) Questa equazione è quella di JAcoßı relativa a $J[y]$.

(2) Determinato a meno di un fattore costante. 
inclusi, sia sfornito di punti coniugati rispetlo alla (4), possiamo allora enunciare il superiore corollario come segue

COROLlaRIo (seconda forma).

Affinchè $J[y]$ abbia minimo in $(G)$ occorre che l'intervallo $\left(x_{1}, x_{2}\right)$, estrewi iuclusi, sia privo di punti coniugati rispetto alla (4).

Questa condizione era nota nella forma diciamo attenuata $\left(^{1}\right)$, cioè che il più piccolo autovalore positivo della $(4)_{\rho}$ fosse maggiore o uguale a uno.

Vedremo nel seguito come la maggiore precisazione apportata con questo corollario permetta la risoluzione completa del problema di estremare $J[y]$ in $(G)$ con procedimento quanto mai semplice.

\section{Le condizioni di trasversalità.}

Dalla relazione (2) deduciamo anche che

per ogni funzione, $g$, di classe $C^{\prime}$ in $\left(x_{1}, x_{2}\right)$, estremamente $J$ in $(G)$ deve aversi

$$
\begin{aligned}
& \int_{x_{1}}^{x_{2}}\left(a g^{\prime} z^{\prime}-\left\{b^{\prime}-c\right\} g z-\left\{p^{\prime}-q\right\} z\right) d x+[z(b g+p)]_{x_{1}}^{x_{2}}=0 \\
& \text { comunque si assegni } z \text {, di classe } C^{\prime} \text {, in }\left(x_{1}, x_{2}\right)
\end{aligned}
$$

dalla quale, con noto procedimento, si trae $\left({ }^{2}\right)$

(1) Cfr. Litehtenstein, 1. c.

$\left({ }^{2}\right)$ Dalla (9), con parziale integrazione per parti si ricava

$$
\int_{x_{1}}^{x_{2}}\left\{a g^{\prime}+\int_{x_{1}}^{x}\left(b^{\prime}-c\right) g+\left(p^{\prime}-p\right) d x\right\} z^{\prime} d x-\left[z \int_{x_{1}}^{x}\left\{\left(b^{\prime}-c\right) g+\left(p^{\prime}-q\right)\right\} d x\right]_{x_{2}}^{x_{1}}+[z(b g+p)]_{x_{1}}^{x_{2}}=0 .
$$

- comunque si assegni $z$ di classe $C^{\prime}-$ da cui, per $z$ nulla agli estremi,

$$
\left.\int_{x_{1}}^{x_{2}}\left\{a g^{\prime}+\int_{x_{1}}^{x}\left(b^{\prime}-c\right) g+p^{\prime}-q\right) d x\right\} z^{\prime} d x=0 .
$$

Segue, pel lemma di Du Bors Reymond,

e però

$$
a g^{\prime}+\int_{x_{1}}^{x}\left(b^{\prime}-c\right) g+\left(p^{\prime}-q\right) d x=\text { cost. } c
$$

$$
\left(a g^{\prime}\right)^{\prime}+\left(b^{\prime}-c\right) g+\left(p^{\prime}-q\right)=0
$$

la quale esprime anche, per essere $a>0$, che $g$ è di classe $C^{\prime \prime}$.

Ma se $g$ soddisfa a questa equazione, dalla (9), si trae: $\left[z\left(a g^{\prime}+b g+p\right)\right]_{x_{1}}^{x_{2}}=0$, comun= que si prenda $z$ di classe $C^{\prime}$, eiò che implica

$(g)^{\prime}$

$$
\left(a g^{\prime}+b g+p\right)_{x_{1}}=\left(a g^{\prime}+b g+p\right)_{x_{1}}=0 .
$$


Condizione necessaria perchè una funzione, $g$, realizzi un estremo di $J$ in $(G)$ è che

(9)

$\left.1^{\circ}\right) g(x)$ sia di classe $C^{\prime \prime}$ in $\left(x_{1}, x_{2}\right)$

$\left.2^{\circ}\right) g(x)$ sia un estremale soddisfacente alle condizioni di trasversalità, e cioè soluzione del sistema seguente

$(9)^{\prime}$

$$
\left\{\begin{array}{l}
\left(a y^{\prime}\right)^{\prime}+\left(b^{\prime}-c\right) y+\left(p^{\prime}-q\right)=0 \\
\left(a y^{\prime}+b y+p\right)_{x_{2}}=\left(a y^{\prime}+b y+p\right)_{x_{1}}=0
\end{array}\right.
$$

II.

\section{Sull'esistenza di valori (eccezionali) del parametro $P$ che rendono possibili sistemi differenziali del tipo.}

$$
\begin{aligned}
& \begin{cases}\left(a z^{\prime}\right)^{\prime}+\rho\left(b^{\prime}-c\right) z=0 & |\rho|>1 \\
{\left[z\left(a z^{\prime}+\rho b z\right)\right]_{x_{1}}^{x_{2}} \geq 0,} & \end{cases} \begin{cases}\left(a z^{\prime}\right)^{\prime}+\rho\left(b^{\prime}-c\right) z=0 & 0<\rho \leq 1 \\
{\left[\left.z\left(a z^{\prime}+\rho b z\right)\right|_{x_{1}} ^{x_{2}} \leq 0,\right.} & \end{cases} \\
&\left\{\begin{array}{l}
\left(a z^{\prime}\right)^{\prime}+\rho \cdot\left(b^{\prime}-c\right) z=0 \\
\left(a z^{\prime}+\rho b z\right)_{x_{1}}=\left(a z^{\prime}+\rho b z\right)_{x_{1}}=0 .
\end{array}\right.
\end{aligned}
$$

I risultati $\left.1 \%, 2 \%, 3^{\circ}\right), 4^{\circ}$ ) del precedente par. I, n. 1 portano alla considerazione di sistemi del tipo (5), $(6),(7)_{1}$, per quel che riguarda i teoremi di esistenza relativi. L' esame di questo problema ë interessante anche indipendentemente da quello che lo ha originato.

Le conclusioni alle quali perverremo, con metodo semplicissimo, consentono di assicurare l'esistenza di soluzioni, diverse da quella identicamente nulla, pei sistemi (5) e (7), e di assegnare alla fine (come conseguenza del nostro studio) una condizione necessaria e sufficiente, di facile applicazione, per l'esistenza di soluzioni del sistema (6). Da oui più in generale si possono conseguire condizioni $\left({ }^{2}\right)$ che consentono di assicurare o escludere l'esistenza di soluzioni di sistemi del tipo $(6)$ e $(7)_{1}$ per valori di $\rho$ contenuti in un intervallo $\left(r_{1}, r_{2}\right)$ assegnato.

Indichiamo a questo scopo con

$$
u(x, \rho) \quad \text { e } \quad v(x, \rho)
$$

(1) L'equaxione che figura in questo sistema $(9)^{\prime}$ è quella, di Eulero, delle estremali relative a $J$, e le condizioni agli estremi $x_{1}$ e $x_{2}$ sono le note condizioni di trasversalità.

(2) Nocessarie e sufficienti. 
due integrali indipendenti dall' equazione

$$
\left(a z^{\prime}\right)^{\prime}+\rho\left(b^{\prime}-c\right) z=0,
$$

per esempio gli integrali determinati, indipendentemente da $\rho$, dalle condizioni iniziali

$(4)_{P}^{\prime}$

$$
\begin{array}{ll}
u\left(x_{1}, \rho\right)=0, & u^{\prime}\left(x_{1}, \rho\right)=1 \\
v\left(x_{1}, \rho\right)=1, & v^{\prime}\left(x_{1}, \rho\right)=0,
\end{array}
$$

e quindi con

$$
z=h u+k v
$$

l'integrale generale della medesima. Abbiamo:

$$
\begin{aligned}
z\left(a z^{\prime}+\rho b z\right) & =(h u+h v)\left\{a \cdot\left(h u^{\prime}+k v^{\prime}\right)+\rho b \cdot(h u+k v)\right\}= \\
& =h^{2}\left(a u u^{\prime}+\rho b u^{2}\right)+h k\left\{a \cdot(u v)^{\prime}+2 \rho b u v\right\}+k^{3}\left(a v v^{\prime}+\rho b v^{2}\right)
\end{aligned}
$$

e quindi

$$
\begin{gathered}
{\left[z\left(a z^{\prime}+\rho b z\right)\right]_{x_{1}}^{x_{2}}=} \\
h^{2}\left[a u u^{\prime}+\rho b u^{2}\right]_{x_{1}}^{x_{2}}+2 h k\left[\frac{1}{2} a \cdot(u v)^{\prime}+\rho b u v\right]_{x_{1}}^{x_{2}}+k^{2}\left[a v v^{2}+\rho b v^{2}\right]_{x_{1}}^{x_{2}} .
\end{gathered}
$$

La possibilità di soddisfare ai sistemi del tipo (5), (6) e $(7)_{1}$, con soluzioni diverse da quella nulla, porta allo studio di questa forma, in $h$ e $k$, per quel che ne riguarda il carattere (cioè l'essere essa definita o semidefinita positiva o negativa, oppure indefiniia).

Pei valori di $\rho$, ad escmpio, pei quali. questa forma riesce definita, sarà impossibile soddisfare al sistema (7), e ad uno dei sistemi (5), (6), etc., etc..

Osserviamo: il carattere di questa forma è indipendente dalla coppia di integrali, $u v$, fissati. Invero pel discriminante, $W(\rho)$, della medesima, tenuto presente che

$$
\left[\frac{1}{2} a(u v)^{\prime}+\rho b u v\right]_{x_{1}}^{x_{2}}=\left[a u v^{\prime}+\rho b u v\right]_{x_{1}}^{x_{2}}=\left[a v u^{\prime}+\rho b v u\right]_{x_{3}}^{x_{2}}
$$

si ha, con evidente significato dei simboli,

$(11)_{1}$

$$
W(\rho)=\left|\begin{array}{ll}
{\left[a u u^{\prime}+\rho b u^{2}\right]_{x_{1}}^{x_{2}}} & {\left[a u v^{\prime}+\rho b u v\right]_{x_{2}}^{x_{2}}} \\
{\left[a v u^{\prime}+\rho b v u\right]_{x_{1}}^{x_{2}}} & {\left[a v v^{\prime}+\rho b v^{2}\right]_{x_{1}}^{x_{2}}}
\end{array}\right|
$$

$\left|\begin{array}{ll}-u_{1} & u_{2} \\ -v_{1} & v_{2}\end{array}\right|\left|\begin{array}{ll}a_{1} u_{1}^{\prime}+\rho b_{1} u_{1} & a_{2} u_{2}^{\prime}+\rho b_{2} u_{2} \\ a_{1} v_{1}^{\prime}+\rho b_{1} v_{1} & a_{2} v_{2}^{\prime}+\rho b_{2} v_{2}\end{array}\right|=\left|\begin{array}{rr}u_{2} & v_{2} \\ -u_{1} & -v_{1}\end{array}\right|\left|\begin{array}{ll}a_{2} u_{2}^{\prime}+\rho b_{2} u_{2} & a_{2} v_{2}^{\prime}+\rho b_{2} a_{2} \\ a_{1} u_{1}^{\prime}+\rho b_{1} u_{1} & a_{1} v_{1}^{\prime}+\rho b_{1} v_{1}\end{array}\right|$.

Quindi, se $\bar{u}, \bar{v}$ è un'altra coppia qualsiasi di integrali indipendenti della 
(4) $)_{\rho}$, pel determinante, $\bar{W}(\rho)$, della corrispondente forma $(10)_{\rho}$ si ottiene, posto che si abbia:

$$
\begin{gathered}
\bar{u}=h_{1} u+k_{1} v ; \quad \bar{v}=h_{2} u+k_{2} v, \\
\bar{W}(\rho)=\left|\begin{array}{ll}
h_{1} & k_{1} \\
h_{2} & k_{2}
\end{array}\right|^{2} W(\rho)\left({ }^{1}\right) .
\end{gathered}
$$

Inoltre pel coefficiente di $h^{2}$ troviamo

$$
\left[a u u^{\prime}+\rho b u^{2}\right]_{x_{1}}^{x_{2}}=h_{1}^{2}\left[a u u^{\prime}+p b u^{2}\right]_{x_{1}}^{x_{2}}+h_{1} k_{1}\left|a(u v)^{\prime}+2 \rho b u v\right|_{x_{1}}^{x_{2}}+k_{1}{ }^{2}\left[a v v^{\prime}+p b v^{2}\right]_{x_{1}}^{x_{2}},
$$

tutto ciò prova l'asserto.

Per lo studio della $(10)_{0}$ possiamo allora prendere, per $u$ e $v$, due integrali che ne rendano l'espressione più semplice.

Supponendo ad esempio che $u$ e $v$ siano gli integrali determinati dalle $(4)_{\rho}{ }^{\prime}$, pel discriminante, $W^{\gamma}(\rho)$, detla $(10)_{\rho}$ otteniamo, in base alla $(11)_{1}$,

$$
W(\rho)=u\left(x_{2}, \rho\right)\left|\begin{array}{ll}
a_{1} & a_{2} u^{\prime}\left(x_{2}, \rho\right)+\rho b_{2} u\left(x_{2}, \rho\right) \\
\rho b_{1} & a_{2} v^{\prime}\left(x_{2}, \rho\right)+\rho b_{2} v\left(x_{2}, \rho\right)
\end{array}\right| .
$$

Ricordiamo ora che all'integrale generale di una qualunque equazione del secondo ordine lineare omogenea si può sempre dare la forma seguente $\left({ }^{2}\right)$

con $\eta$ e $\xi$ funzioui,

$$
z=e^{n}(h \cos \xi+k \operatorname{sen} \xi)
$$

$$
\eta=\eta(x, \rho) \text { e } \xi=\xi(x, \rho),
$$

continue soddifacenti a queste condizioni

(14) $\left\{\begin{array}{c}\eta\left(x_{1}, \rho\right)=\eta\left(x_{1}, \rho\right)=0, \quad \xi\left(x_{1}, \rho\right)=0, \quad \xi\left(x_{1}, \rho\right)=1, \\ \text { e, per ogni } p \text {, inoltre con } \\ \xi(x, \rho) \text { fnnzione di } x \text { a derivata sempre positiva. }\end{array}\right.$

Pertanto

$$
u=e^{n} \operatorname{sen} \xi \quad v=e^{n} \cos \xi
$$

e sostituendo nella (11)

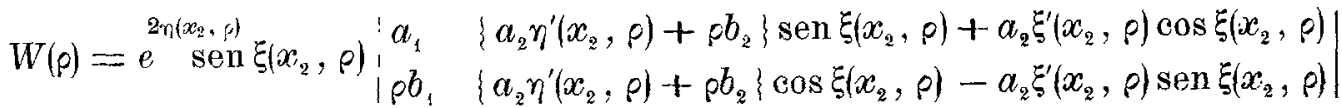

$$
\begin{aligned}
& =e^{\ln \left(x_{2}, \rho\right)} \operatorname{sen} \xi\left(x_{2}, \rho\right)\left|\begin{array}{rr}
a_{1} & \cos \left\{\xi\left(x_{2}, \rho\right)-\gamma\right\} \\
\rho b_{3} & -\operatorname{sen}\left\{\xi\left(x_{2}, \rho\right)-\gamma\right\}
\end{array}\right| \sqrt{\left\{a_{2} \eta^{\prime}\left(x_{2}, \rho\right)+\rho b_{2}\right\}^{2}+\left\{a_{2} \xi^{\prime}\left(x_{2}, \rho\right)\right\}^{2}} \\
& \text { (1) Si noti che }\left|\begin{array}{ll}
h_{1} & k_{1} \\
h_{2} & k_{2}
\end{array}\right| \neq 0 \text {. }
\end{aligned}
$$


avendo posto

$$
\gamma=\operatorname{arc}\left\{\begin{array}{l}
\cos \frac{a_{2} \xi^{\prime}\left(x_{2}, \rho\right)}{\sqrt{\left\{a_{2} \eta^{\prime}\left(x_{2}, \rho\right)+\rho b_{2}\right\}^{2}+\left\{a_{2} \xi^{\prime}\left(x_{2}, \rho\right)\right\}^{2}}} \\
\operatorname{sen} \frac{a_{2} \eta^{\prime}\left(x_{2}, \rho\right)+\rho b_{2}}{\sqrt{\left\{a_{2} \eta^{\prime}\left(x_{2}, \rho\right)+\rho b_{2}\right\}^{2}+\left\{a_{2} \xi^{\prime}\left(x_{2}, \rho\right)\right\}^{2}}},
\end{array}\right.
$$

$\gamma$ risulta funzione, $\gamma=\gamma(\rho)$, di $\rho$ determinata a meno di multipli di $2 \pi$.

Fra gli infiniti valori di $\gamma$ associati a un fissato valore, $\bar{\rho}$, di $\rho$ fissamone uno, ad esempio quello, $\bar{\gamma}$, di modulo minimo e conveniamo poi, per la scelta del valore corrispondente a ogni altro valore di $\rho$, di adottare il criterio per il qnale sia conservata la conlinuità di $\gamma(\rho)$.

In questa guisa $\gamma$ risulta funzione uniforme e continua di $\rho$ limitata al variare di questo parametro, chè il relativo coseno - a causa delle (14) e (16) si mantiene sempre positivo. Dovrà aversi anzi

$$
-\frac{\pi}{2}<\gamma<\frac{\pi}{2}
$$

Abbiamo ancora (con evidente significato dei simboli)

$$
\begin{aligned}
W(\rho) & =-e^{2 \gamma_{2}} \sqrt{\left\{a_{2} \eta_{2}{ }^{\prime}+\rho b_{2}\right\}^{2}+\left\{a_{2} \xi_{2}\right\}^{2}}\left\{a_{1} \operatorname{sen}\left(\xi_{2}-\gamma\right)+\rho b_{1} \cos \left(\xi_{2}-\gamma\right)\right\} \operatorname{sen} \xi_{2} \\
& =-e^{2 \gamma_{2}} \sqrt{\left\{a_{2} \eta_{2}^{\prime}+\rho b_{2}\right\}^{2}+\left\{a_{2} \xi_{2}^{\prime}\right\}^{2}} \sqrt{\left\{a_{1}^{2}+\rho^{2} b_{1}^{2}\right.} \operatorname{sen}\left(\xi_{2}-\gamma+\gamma_{1}\right) \operatorname{sen} \xi_{z} \equiv \\
& \equiv-e^{\alpha(\rho)} \operatorname{sen} \xi_{2} \operatorname{sen}\left(\xi_{2}-\omega\right)
\end{aligned}
$$

dove,

$$
\gamma_{1}=\operatorname{arc}\left\{\begin{array}{l}
\cos \frac{a_{1}}{\sqrt{a_{1}^{2}+\rho^{2} b_{1}^{2}}} \\
\operatorname{sen} \frac{\rho_{1} b_{1}}{\sqrt{a_{1}^{2}+\rho^{2} b_{1}^{2}}}
\end{array}\right.
$$

può considerarsi (poichè $a_{1}>0$ ), come $\gamma$, limitata e compresa fra $-\frac{\pi}{2}$ e $\frac{\pi}{2}$. Pertanto

$$
\omega \equiv \gamma-\gamma_{1}
$$

risulta continua e soddisfacente alla limitazione

$$
-\pi<\omega<\pi \text {. }
$$

Medesimamente troviamo per il primo coeffciente della $(10)_{q}$

$$
\begin{gathered}
{\left[u\left(a u^{\prime}+\rho b u\right)\right]_{x_{1}}^{x_{2}}=u_{2}\left(a_{2} u_{2}^{\prime} \rho b_{2} u_{2}\right)=} \\
=e^{2 r_{2} 2} \sqrt{\left(a_{2} \eta_{2}^{\prime}+\rho b_{2}\right)^{2}+\left(a_{2} \bar{\xi}_{2}^{\prime}\right)^{2}} \operatorname{sen} \xi_{2} \cos \left(\xi_{z}-\gamma\right)
\end{gathered}
$$


dove $\gamma$ è sempre definito dalle (16) etc.. Dalla noterole forma

$$
W(\rho)=e \cdot \operatorname{sen} \xi_{2} \operatorname{sen}\left(\omega-\xi_{2}\right)
$$

data al discriminante della $(10)_{\beta}$ deduciamo immediatamente l'esistenza di infiniti valori (eccezionali) di $\rho$ che annullano $W(o)$, anzi che annullano separatamente $\mathrm{i}$ due determinanti componenti, in base alla $(11)_{1}, W(\rho)$ stesso (1).

Infatti, relativamente alla $(4)_{p}$ sussiste, com'è noto $\left({ }^{2}\right)$, un teorema d'oscillazione, per il quale se $\left(b^{\prime}-c\right)$ ha tratti positivi (negativi) il numero di zeri di ogni integrale della $(4)_{0}$ stessa, quindi in particolare di

$$
u=e^{n} \operatorname{sen} \xi(x, \rho) \text {, }
$$

contenuti in $\left(x_{1}, x_{2}\right)$ cresce indefinitamente col crescere indefinito di $p$ per valori positivi (negativi).

Ma il minimo numero, $n$, di zeri dell' integrale (18) è dato (in base alla (14)) $\left({ }^{2}\right) \mathrm{da}$

$$
n=\left[\frac{\xi\left(x_{2}, \rho\right)}{\pi}\right]+1
$$

ne consegue che al divergere di $\rho$ (per valori positivi o per valori negativi)

$$
\xi\left(x_{2}, p\right) \rightarrow+\infty
$$

e medesimamente, grazie alla $(17)_{1}$,

$$
\xi\left(x_{2}, \rho\right)-\omega \rightarrow+\infty
$$

da cui, a causa della continuità di $\xi\left(x_{2}, \rho\right)$ e di w(p), si deduce l'esistenza di infiniti valori (eccezionali) di $p$ che annullano $W(\rho)$.

Più precisamente segue l'esistenza di infiniti valori reali di $p$, aventi l' infinito come punto limite, che soddisfano alla equazione

$$
\left|\begin{array}{ll}
u\left(x_{1}, \rho\right) & u\left(x_{2}, \rho\right) \\
v\left(x_{1}, \rho\right) & v\left(x_{2}, \rho\right)
\end{array}\right| \equiv H \operatorname{sen} \xi\left(x_{2}, \rho\right)=0
$$

e di infiniti valori di $\rho$, distinti, in generale $\left({ }^{3}\right)$, dai primi, etc. etc., soddisfacenti a quest' altra equazione

$$
\left|\begin{array}{ll}
a_{1} u^{\prime}\left(x_{1}, \rho\right)+\rho b_{1} u\left(x_{1}, \rho\right) & a_{2} u^{\prime}\left(x_{2}, \rho\right)+\rho b_{2} u\left(x_{2}, \rho\right) \\
a_{1} v^{\prime}\left(x_{1}, \rho\right)+\rho b_{1} v\left(x_{1}, \rho\right) & a_{2} v^{\prime}\left(x_{2}, \rho\right)+\rho q_{2} v\left(x_{2}, \rho\right)
\end{array}\right| \equiv V \operatorname{sen}\left\{\xi\left(x_{2}, \rho\right)-\omega(\rho)\right\}
$$

con $H$ e $V$ avendo designato fattori non nalli dipendenti da $\rho$.

(1) I due fattori non esponenziali che figurauo nell' ultimo membro della (17) sono pro. porzionali al primo e al secondo determinante che figurano nella (11) .

(2) Cfr. G. Mammana, l. c. art. 30 e seguenti.

(3) Notiamo che $\omega(\rho)=\gamma(\rho)-\gamma_{1}(\rho)$ dipende da $b_{1}$ mentre $\xi\left(x_{2}, \rho\right)$ puô considerarsi indi. pendente da questo coefficiente. 
Da tutto ciò si deduce, tenuto anche conto della (17)' e del fatto che la trascendente, intera in $\rho$,

$$
u\left(x_{2}, \rho\right)=e^{\gamma_{2}} \operatorname{sen} \xi\left(x_{2}, \rho\right)
$$

è priva di punti multipli $\left({ }^{1}\right)$, il

Teorema II. - Esistono infiniti valori di $\rho$, aventi l'infinito come punto limite che rendono la forma $(10)_{\rho}$ definita, semidefinita, indefinita.

Ne consegue il

Teonema III. - Esistono infiniti valori eccezionali di $\rho$, aventi l'infinito come punto limite, pei quali è possibile soddisfare ai sistemi

$\left\{\begin{array}{l}\left(a z^{\prime}\right)^{\prime}+\rho\left(b^{\prime}-c\right) z=0 \\ {\left[z\left(\alpha z^{\prime}+\rho b z\right)\right]_{x_{1}} x^{x_{2}} \geq 0,}\end{array} \quad\left\{\begin{array}{l}\left(\alpha z^{\prime}\right)^{\prime}+\rho\left(b^{\prime}-c\right) z=0 \\ \left(\alpha z^{\prime}+\rho b z\right)_{x_{2}}=\left(a z^{\prime}+\rho b z\right)_{x_{2}}=0,\end{array} \quad\left\{\begin{array}{l}\left(a z^{\prime}\right)^{\prime}+\rho\left(b^{\prime}-c\right) z=0 \\ (z)_{x_{2}}=(z)_{x_{1}}=0,\end{array}\right.\right.\right.$

con soluzioni non nulle identicamente.

(1) Che gli zeri di $u\left(x_{2}, \rho\right)$ e quindi di $\operatorname{sen} \xi\left(x_{2}, \rho\right)$ siano tutti semplici si può dedurre da quoste semplicissime considerazioni. Poichè $u(x, \rho)$ è la soluzione della (t) nulla in $x_{1}$ e ivi a derivata uguale ad uno, nell' ipotesi che $\bar{\rho}$ sia zero di $u\left(x_{2}, \rho\right)$, dalla identità

$$
\{a u(x, p)\}^{\prime}+p \cdot\left(b^{\prime}-c\right) u(x, p)=0
$$

derivando rapporto a $p$, deduciamo, posto $w=\frac{\partial u}{\partial \rho}$,

$$
\left(a w^{\prime}\right)^{\prime}+p \cdot\left(b^{\prime}-c\right) w+\left(b^{\prime}-c\right) u=0, \quad \text { con } \quad w\left(x_{1}, \rho\right)=w^{\prime}\left(x_{1} ; \rho\right)=0,
$$

da olli, per $p$ uguale a $\rho$, si trae simultaneamente

(20) $\left(a u^{\prime}\right)^{\prime}+\bar{\rho} \cdot\left(b^{\prime}-c\right) u=0, \quad\left(a w^{\prime}\right)^{\prime}+\overline{\bar{\rho}} \cdot\left(b^{\prime}-c\right) w+\left(b^{\prime}-c\right) u=0, \quad u\left(x_{1}, \bar{\rho}\right)=u\left(x_{2}, \bar{\rho}\right)=v\left(x_{1}, \bar{\rho}\right)=0$,

Ora supponiamo $\bar{\rho}$ zero multiplo di $u\left(x_{2}, \rho\right)$, e ciò̀: $\left(\frac{\partial u}{\partial \rho}\right)_{\rho}=w\left(x_{2}, \bar{\rho}\right)=0$,

Dalle $(20)$ si ricara: $\left(a u^{\prime}\right)^{\prime} v-\left(a v^{\prime}\right)^{\prime} u=\left(b^{\prime}-c\right) u^{2}$, da cui

$$
\left[w a u^{\prime}\right]_{x_{1}}^{x_{2}}-\left[u a w^{\prime}\right]_{x_{1}}^{x_{2}}=0=\int_{x_{1}}^{x_{2}}\left(b^{\prime}-c\right) u^{2} d x
$$

D'altra parte dalla prima delle (20) si deduce pure

quindi

$$
\left[u\left(a u^{\prime}\right)\right]_{x^{3}}^{x_{2}}-\int_{x^{\mathrm{L}}}^{x_{2}} a u^{\prime 2} d x=-\int_{x_{1}}^{x_{2}} a u^{\prime 2} d x=-\rho \int_{x_{1}}^{x_{2}}\left(b^{\prime}-c\right) u^{2} d x
$$

cio ohe è assurdo.

$$
\int_{x_{1}}^{x_{2}} a e^{\prime 2} d x=0
$$


Quanto ai sistemi

$$
\left\{\begin{array} { l } 
{ ( a z ^ { \prime } ) ^ { \prime } + 0 \cdot ( b ^ { \prime } - c ) z = 0 } \\
{ [ z ( a z ^ { \prime } + \rho b z ) ] _ { x _ { 1 } } ^ { ( x _ { z } } \leq 0 , }
\end{array} \quad \left\{\begin{array}{l}
\left(a z^{\prime}\right)^{\prime}+\rho \cdot\left(b^{\prime}-c\right) z=0 \\
\left.\left(a z^{\prime}+\rho b z\right)_{x_{2}}+a z^{\prime}+\rho b z\right)_{x_{1}}=0
\end{array} \quad \text { con } 0<p \leq 1,\right.\right.
$$

vedremo più avanti che $\dot{e}$ possibile assegnare, come conseguenza di un teorema di calcolo delle variazioni, un criterio, di applicazione immediata, che permette assicurare o escludere l'esistenza di soluzioni diverse da quella nulli.

Ossenvazione. - Le considerazioni fatte nel presente paragrafo si riferiscono ad equazioni, come la $(4)_{\rho}$, col coefficiente della funzione incognita lineare e omogeneo in $\rho$. Ma i risultati raggiunti si estendono anche al caso in cui questo coefficiente didenda comunque da $\rho\left({ }^{1}\right)$, purchè ptr la corri. spondente equazione sussista un teorema di oscillazione, quindi in particolare quando detto coetficiente sia razionale in $p^{\circ}$

Da ciò si desume la possibilità di ottenere, come accennammo in principio, teoremi di esistenza di valori eccezionali contenuti in un intervallo assegnato $\left(r_{1}, r_{2}\right)$, relativi a sistemi del tipo considerato con una condizione quadratica in due punti, o due condizioni lineari agli estremi etc. etc..

III.

\section{Condizione necessaria e sufficiente per l'esistenza di minimo di $J[y]$ in $(G)$.}

Secondo dunque quanto abbiamo constatato, perchè il funzionale $J[y]$ ammetta minimo in $(G)$ occorre che

ж) esista un estremale soddisfacente alle condizioni di trasversalita, e cioè una soluzione del sistema $(9)^{\prime}$;

3) non sia possibile soddisfare, con soluzioni diverse da quella identieamente nulla, ad alcuno dei due sistemi $(6)_{1}$ e $(6)_{2}$ di cui al teorema $\left.1, b\right)$;

$\gamma)$ ove esista una soluzione, non nulla identicamente, del sistema (7), essa sia anche soluzione del sistema differenziale (con tre condizioni lineari agli estremi) (8).

Dalle superiori condizioni $\beta$ e $\gamma$ ) consegue, in base ai risultati conseguiti nel precedente paragrafo secondo, e posto che $u$ e $v$ rappresentino due integrali indipendenti della $(4)_{\rho}$, il

(1) Cfr. G. Mammana, 1. c. 
Teorema IV. - Affinchè $J[y]$ sia fornito di minimo in $(G)$ occorre che la forma, in $h$ e $k$,

$(10)_{\rho} \quad h^{2}\left[u\left(a u^{\prime}+p b u\right)\right]_{x_{1}}^{x_{2}}+2 h k\left[\frac{1}{2} a(u v)^{\prime}+\left.\rho b u v\right|_{x_{1}} ^{x_{2}}+k^{\nu}\left[v\left(a v^{\prime}+\rho b v\right)\right]_{x_{2}}^{x_{2}} \quad 0<\rho \leq 1\right.$

sia definita o semidefinita positiva.

Ne consegue (da questo teorema e dal corollario del precedente teorema I) in particolare il

Teorema V (prima forma). - Condizione necessaria perchè il funzionale $J[y]$ abbia minimo in $(G)$ è che

$$
\begin{aligned}
& \text { (a) l'intervallo }\left(x_{1}, x_{2}\right) \text {, estremi inclusi, sia privo di punti co- } \\
& \left(a z^{\prime}+b z\right)_{x_{2}}=\left(a z^{\prime}+b z\right)_{x_{1}}=[p z]_{x_{1}}^{x_{2}}-\int_{x_{1}}^{x_{2}}\left(p^{\prime}-q\right) z d x=0 .
\end{aligned}
$$

Questa condizione (8) implica la possibilità di soddisfare, con valori non entrambi nulli, al sistema, in $h$ e $k$, seguente

$$
\begin{aligned}
& h\left(a u^{\prime}+b u\right)_{x_{2}}+k\left(a v^{\prime}+b v\right)_{x_{2}}=0 \\
& h\left(a u^{\prime}+b u\right)_{x_{2}}+k\left(a v^{\prime}+b v\right)_{x_{2}}=0 \\
& h\left[p u-\int_{x_{2}}^{x}\left(p^{\prime}-q\right) u d x\right]_{x_{1}}^{x_{2}}+k\left[p v-\int_{x_{2}}^{\infty}\left(p^{\prime}-q\right) v d x\right]_{x_{1}}^{x_{2}}=0
\end{aligned}
$$

sistema equivalente a quest'altro - a causa della (11), e della superiore condizione a) -

$$
\begin{aligned}
& h\left[u\left(a u^{\prime}+b u\right)\right]_{x_{1}}^{x_{2}}+h\left[v\left(a u^{\prime}+b u\right)\right]_{x_{1}}^{x_{2}}=0 \\
& h\left[u\left(a v^{\prime}+b v\right)\right]_{x_{1}}^{x_{2}}+k\left[v\left(\alpha v^{\prime}+b v\right)\right]_{x_{1}}^{x_{2}}=0 \\
& h\left[p u-\int_{x_{1}}^{x}\left(p^{\prime}-q\right) u d x\right]_{0_{1}}^{x_{2}}+k\left[\left.p v \int_{x_{1}}^{x}\left(p^{\prime}-q\right) v d x\right|_{x_{1}} ^{x_{3}}=0 .\right.
\end{aligned}
$$


Si ha pertanto il

Teorema $V$ (seconda forma). - Perchè $J[y]$ abbia minimo in $(G)$ occorre

a) l'intervallo $\left(x_{1}, x_{2}\right)$, estremi inclusi, sia privo di punti coniugati rispetto alla $(4)$,

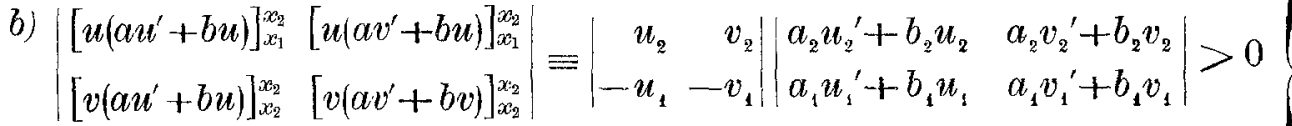

$$
\begin{aligned}
& {\left[u\left(a u^{\prime}+b u\right)\right]_{x_{1}}^{x_{2}}>0}
\end{aligned}
$$

con $u$ e $v$ avendo designato due integrali indipendenti qualsiansi della (4),

oppure

a) l'intervallo $\left(x_{1}, x_{2}\right)$, estremi inclusi, sia privo di punti coniugati rispetto alla (4)

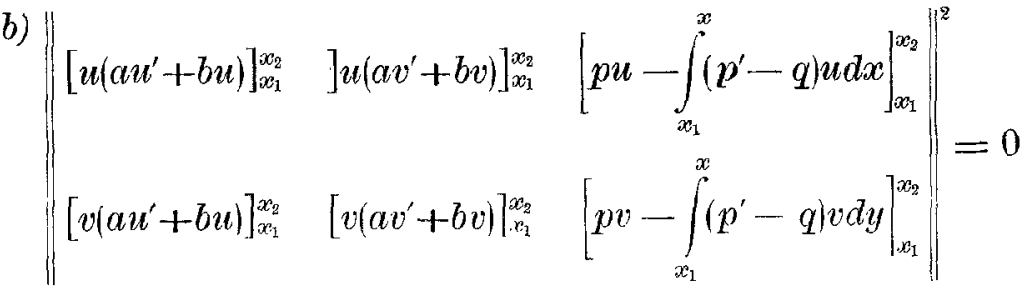

$$
\begin{aligned}
& {\left[u\left(a u^{\prime}+b u\right)\right]_{x_{2}}^{x_{1}} \geq 0, \quad\left[v\left(a v^{\prime}+b v\right)\right]_{x_{1}}^{x_{2}} \geq=0 .}
\end{aligned}
$$

La precisazione apportata col corollario del teorema I rende possibile invertire - e in maniera quanto mai semplice - questo teorema. Si ha il

TeOREMa VI. - Le superiori condizioni a) e b) - necessarie per l'esistenza di minimo di J in (G) - sono anche sufficienti.

Il procedimento che seguiremo per la dimostrazione di questo teorema, permette altresi di ritrovare ancora le condizioni a) e b) come necessarie, conseguendo così un risultato notevole della teoria delle autofunzioni.

Si dà cioè un teorema che, estendendone uno classico sugli antovalori, assegna, sotto forma semplice ed espressiva, una condizione che è necessaria e sufficiente per l'esistenza di valori eccezionali - contenuti nell'intervallo $(0,1)$ - relativi a una equazione lineare,

$$
\left(a u^{\prime}\right)^{\prime}+\rho Q u=0
$$

con una condizione quadratica del tipo

$$
\left[u\left(a u^{\prime}+\rho b u\right)\right]_{x_{1}}^{x_{2}} \geq 0
$$

o con due condizioni lineari come queste

$$
\left(a u^{\prime}+\rho b u\right)_{x_{1}}=\left(a u^{\prime}+\rho b u\right)_{x_{2}}=0,
$$


teorema suscettibile di estensione al caso ohe l'intervalto sia qualsiasi $\left(r_{1}, r_{2}\right)$ e il coefficiente di $u$ non lineare omogeneo in $\rho$ ma dipendente comunque da questo parametro (1).

Dry. Teorema VI. - Supponiamo soddisfatta la condizione a) di cui al superiore teorema e ciò̀ che l'intervallo $\left(x_{1}, x_{2}\right)$, estremi inclusi, sia privo di punti coningati rispetto alla (4). In quest'ipotesi, se $u$ e $v$ sono integrali indipendenti qualsiansi della (4), il determinante

$$
\left|\begin{array}{ll}
u\left(x_{1}\right) & u\left(x_{2}\right) \\
v\left(x_{1}\right) & v\left(x_{2}\right)
\end{array}\right| \neq 0
$$

Consideriamo il sistema integrale fondamentale della (4), $\bar{u}, \bar{v}$, associato alle seguenti condizioni agli estremi

(4), $\quad \begin{cases}\bar{u}\left(x_{1}\right)=0 \\ \vec{u}\left(x_{2}\right)=1, & (4)_{2}\end{cases}$

- sistema perfettamente determinato a causa della (22) - e l'integrale, $\bar{w}$, dell' equazione d' EULER

$$
\left(\alpha y^{\prime}\right)+\left(b^{\prime}-c\right) y+\left(p^{\prime}-q\right)=0,
$$

univocamente determinato, per la (22), dalle condizioni agli estremi

$$
\bar{w}\left(x_{1}\right)=\bar{w}\left(x_{2}\right)=0 \text {. }
$$

Per l'integrale generale di quest' ultima equazione abbiamo allora (indicando con $y_{1}$ e $y_{2}$ costanti arbitrarie)

$$
y=y_{1} \bar{v}+y_{2} \bar{u}+\bar{w} \text {. }
$$

Si ha, grazie alla particolare scelta di $\bar{u}, \bar{v}$ e $\bar{w}$,

$$
y\left(x_{1}\right)=y_{1} ; \quad y\left(x_{2}\right)=y_{2} .
$$

La (24) pertanto rappresenta l'estremale, ben determinata, passante pei punti

$$
P_{1} \equiv\left\{x_{1}, y_{1}\right] \text { e } P_{2} \equiv\left[x_{2}, y_{2}\right]
$$

Questa estremale, univocamente determinata comunque si assegnino $P_{1}$ e $P_{2}$ sulle rette $x=x_{1}$ e $x=x_{2}$, realizza, com' ̀̀ ben noto, nella superiore ipotesi, il minimo assoluto proprio di $J[y]$ nell'insieme, $\left(G_{y_{1} y_{2}}\right)$, di tutte le curve di $(G)$ passanti per $P_{1}$ e $P_{2}$.

(1) Purchè relativamente alla equazione (1) sussista un teorema di oscillızione. Cfr. G. Mammana, l, c. 
Ora, se $J[y]$ è fornito di minimo in $(G)$, per ogni minimante, $\bar{y}(x)$, di questo funzionale si ha anche, ovviamente,

$$
\bar{y}(x) \text { è minimante di } J[y] \text { in }\left(G_{\bar{y}\left(x_{1}\right), \bar{y}\left(x_{2}\right)}\right),
$$

inoltre l'estremale $y=\bar{y}(x)$, passante pei punti

$$
\left[x_{1}, \bar{y}\left(x_{1}\right)\right], \quad\left[x_{2}, \bar{y}\left(x_{2}\right)\right]
$$

realizza il minimo assoluto proprio di $J[y]$ nell'insieme $(E)$ (delle estremali) rappresentato dalla (24).

Viceversa, un eventuale estremale, $y=y(x)$, minimante $J[y]$ nell' in sieme (E) realizza - pel fatto che ogni estremale $y=y_{2} \bar{u}+y_{1} \bar{v}+\bar{w}$, minimizza $J$ in $\left(G_{y_{2} y_{0}}\right)$ - il minimo assoluto di $J[y]$ in $(G)$.

Pertanto il funzionale $J[y]$ ha minimo in $(G)$ allora e solo quando: l'intervallo $\left(x_{1}, x_{2}\right)$ è privo di punti coniugati rispetto all' equazione di JABOBI (4), e inoltre lo stesso funzionale è fornito di minimo nell'insieme, $(E)$, delle relative estremali; cioè è fornita di minimo la funzione quadratica, in $y_{1}$ e $y_{z}$, seguente

$$
J\left[y_{2} \bar{u}+y_{1} \bar{v}+\bar{w}\right]
$$

Il posto problema di calcolo delle variazioni è cosi ricondotto, grazie alla precisnzione apportata col cocollario del teorema I, a un banale problema di ricerca di minimo di una funzione quadratica in due variabili.

Notiamo poi che se $u$ e $v$ sono integrali indipendenti qualsiansi dell'equazione di JАCOBI (4), e $w$ un qualsivoglia integrale della (23), la classe di estremali rappresentata dallá combinazione, a coefficienti, $g_{1}$ e $g_{2}$, costanti (arbitrarie)

$$
y=g_{1} u+g_{2} v+w \equiv g
$$

coincide, salvo l'ordine degli elementi, con la classe di estremali (24).

Sono partanto identici i problemi della ricerca di minimo di

$$
J\left[y_{1} \bar{v}+y_{2} \vec{u}+\vec{w}\right] \text { e di } J\left[g_{1} u+g_{3} v+w\right]
$$

Ma perchè la funzione quadratica, in $g_{1}$ e $g_{2}$,

$$
J[g]=J\left[g_{1} u+g_{2} v+w\right]
$$

abbia minimo, occorre e basta che la forma quadratica ad essa associata sia definita positiva, oppure semidefinita positiva purchè allora contemporaneamente siano compatibili le equazioni, lineari in $g_{1}$ e $g_{2}$, che si ottengono eguagliando a zero la derivate parziali di $J[g]$ rapporto a $g_{1}$ e $g_{2}$. 
In simboli

$J[g]$ è fornita di minimo in $(G)$ allora e solo quando

$$
\left.\mid \begin{array}{c}
H \equiv\left|\begin{array}{cc}
\frac{\partial^{2} J}{\partial g_{1}^{2}} & \frac{\partial^{2} J}{\partial g_{1} \partial g_{2}} \\
\frac{\partial^{2} J}{\partial g_{1} \partial g_{2}} & \frac{\partial^{2} J}{\partial g_{2}^{2}}
\end{array}\right|>0, \quad \frac{\partial^{2} J}{\partial g_{1}^{2}}>0 \\
\text { oppure } \\
H=0, \quad \frac{\partial^{2} J}{\partial g_{1}^{2}} \geq 0, \quad \frac{\partial^{2} J}{\partial g_{2}^{2}} \geq 0 \\
\text { e risultino compatibili le equazioni } \\
\frac{\partial J}{\partial g_{1}} \equiv \int_{x_{1}}^{x_{2}}\left(a g^{\prime} u^{\prime}+b(g u)^{\prime}+c g u+p u^{\prime}+q u\right) d x=0 \\
\frac{\partial J}{\partial g_{2}} \equiv \int_{x_{1}}^{x_{2}}\left(a g^{\prime} v^{\prime}+b(g v)^{\prime}+c g v+p v^{\prime}+q v\right) d x=0
\end{array}\right\}{ }_{(25)_{2}}^{(25)_{2}}
$$

Le $(25)_{1}$ e $(25)_{2}$ si esprimono semplicemente mediante i valori agli estremi di $u, v$ e $w$ e delle rispettive derivate, ove si tenga presente che $u$ e $v$ sono soluzioni della (4) e $w$ della (23).

Si ha

$$
\begin{aligned}
& \frac{\partial J}{\partial g_{1}} \equiv\left[u\left(a g^{\prime}+b g+p\right)\right]_{x_{1}}^{x_{2}}-\int_{x_{1}}^{x_{2}} u\left\{\left(a g^{\prime}\right)^{\prime}+\left(b^{\prime}-c\right) g+\left(p^{\prime}-q\right)\right\} d x= \\
& =\left[u\left(a g^{\prime}+b g+p\right)\right]_{x_{1}}^{x_{2}}=\left\{u\left(a g^{\prime}+b g+p\right)\right\}_{x_{2}}-\left\{u\left(a g^{\prime}+b g+p\right)\right\}_{x_{1}}= \\
& =g_{1}\left[u\left(a u^{\prime}+b u\right)\right]_{x_{1}}^{x_{2}}+g_{2}\left[u\left(a v^{\prime}+b v\right)\right]_{x_{1}}^{x_{2}}+\left[u\left(a v^{\prime}+b w+p\right)\right]_{x_{1}}^{x_{x_{2}}}
\end{aligned}
$$

medesimamente

$$
\begin{gathered}
\frac{\partial J}{\partial g_{2}} \equiv\left[v\left(a g^{\prime}+b g_{1}+p\right)\right]_{x_{1}}^{x_{2}}= \\
=g_{1}\left[v\left(a u^{\prime}+b u\right)\right]_{x_{1}}^{x_{2}}+g_{z}\left[v\left(a v^{\prime}+b v\right)\right]_{x_{1}}^{x_{2}}+\left[v\left(a w^{\prime}+b v^{\prime}+p\right)\right]_{x_{x_{1}}}^{x_{x_{2}}} .
\end{gathered}
$$

Segue, da queste relazioni, che il sistema $(25)_{2}$ è equivalente, in base alla (22), a quest'altro

$$
\left\{\begin{array}{l}
\left(a g^{\prime}+b g+p\right)_{x_{2}}=\left(a_{2} u_{2}^{\prime}+b_{2} u_{2}\right) g_{1}+\left(a_{2} v_{2}{ }^{\prime}+b_{2} v_{2}\right) g_{2}+\left(a_{2} v_{2}^{\prime}+b_{2} w_{2}+p_{2}\right)=0 \\
\left(a g^{\prime}+b g+p\right)_{x_{1}}=\left(a_{1} u_{1}^{\prime}+b_{1} u_{1}\right) g_{1}+\left(a_{1} v_{1}^{\prime}+b_{1} v_{1}\right) g_{2}+\left(a_{1} v_{1}^{\prime}+b_{1} w_{1}+p_{1}\right)=0
\end{array}\right.
$$

che coincide col sistema $(9)^{\prime}$ delle condizioni di trasversalità. 
Troviamo pure, poichè

$$
\begin{gathered}
\frac{\partial^{2} J}{\partial g_{1}^{2}}=\left[u\left(a u^{\prime}+b u\right)\right]_{x_{1}}^{x_{2}}, \quad \frac{\partial^{2} J}{\partial g_{1} \partial g_{2}}=\left[u\left(\left(w v^{\prime}+b v\right)\right]_{x_{1}}^{x_{2}}=\left[v\left(a u^{\prime}+b u\right)\right]_{x_{1}}^{x_{2}},\right. \\
\frac{\partial^{2} J}{\partial g_{2}^{2}}=\left[v\left(a v^{\prime}+b v\right)\right]_{x_{1}}^{x_{2}},
\end{gathered}
$$

chè la forma quadratica, in $g_{1} \otimes g_{2}$,

$$
g_{1}^{2}\left[u\left(a u^{\prime}+b u\right)\right]_{x_{1}}^{x_{2}}+2 g_{1} g_{2}\left[u\left(a v^{\prime}+b v\right)\right]_{x_{1}}^{x_{2}}+g_{2}^{2}\left[v\left(a v^{\prime}+b v\right)\right]_{x_{1}}^{x_{2}},
$$

associata alla funzione $J[g]$ è identica alla forma (20) di cui al precedente teorema V.

Concludiamo quindi che:

Condizione necessaria e sufficiente affinchè il funzionale $J$ abbia minimo in $(G)$ è che l'intervallo $\left(x_{1}, x_{2}\right)$ sia privo di punti coniugati rispetto all' equazione di J

$$
\left\{\begin{array}{c}
H \equiv\left|\begin{array}{cc}
{\left[u\left(a u^{\prime}+b u\right)\right]_{x_{1}}^{x_{2}}} & {\left[u\left(a v^{\prime}+b v\right)\right]_{x_{1}}^{x_{2}}} \\
{\left[v\left(a u^{\prime}+b u\right)\right]_{x_{1}}^{x_{2}}} & {\left[v\left(a v^{\prime}+b v\right)\right]_{x_{1}}^{x_{2}}}
\end{array}\right| \equiv\left|\begin{array}{rr}
u_{2} & v_{2} \\
-u_{1} & -v_{1}
\end{array}\right|\left|\begin{array}{ll}
a_{2} u_{2}^{\prime}+b_{2} u_{2} & a_{2} v_{2}^{\prime}+b_{2} v_{2} \\
a_{1} u_{1}^{\prime}+b_{1} u_{1} & a_{1} v_{1}^{\prime}+b_{1} v_{1}
\end{array}\right|>0 \\
{\left[u\left(a u^{\prime}+b u\right)\right]_{x_{1}}^{x_{2}}>0}
\end{array}\right.
$$

oppure

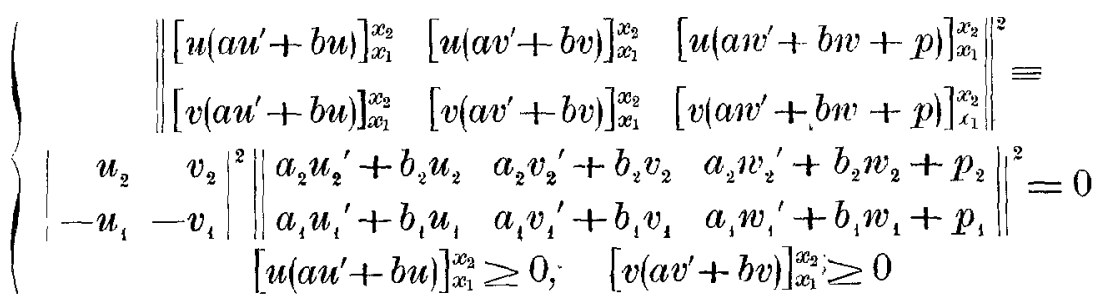

dove con $u$ e $v$ abbiamo designato due integrali indipendenti dell' equazione di JACOBI (4) e con $w$ un integrale qualsiasi dell' equazione di EuLERo (23).

Nell'ipotesi (26) esiște un'unica estremale soddisfacente alle condizioni di trasversalità $(9)^{\prime}$ la quale realizza il minimo assoluto proprio di $J[y]$ in $(G)$.

Nell'ipotesi (26), invece vi sono infinite estremali (una semplice infinità) soddisfacenti alle condizioni di trasversalita, ciascuna delle quali realiza il minimo assoluto di $J[y]$ in $(G)$.

La condizione (26) coincide con la (21) del teorema $V$, e la (26), equivale, come verificheremo subito, alla (21), il nostro teorema VI è dunque dimostrato.

NотA. - Le condizioni (26) e (21), formalmente diverse sono equivalenti, cio segue dalle considerazioni seguenti: 
Indichiamo con

(m)

$$
M_{12}, M_{13}, M_{23},
$$

i minori di ordine massimo della matrice $(26)_{1}$ formati rispettivamente con le colonne prima e seconda, prima e terza, seconda e terza, e con

$$
N_{12}, N_{13}, N_{23} \text {, }
$$

i corrispondenti minori della matrice $(21)_{1}$.

L'annullarsi della (26) implica

$(\mathrm{m})$

$$
M_{12}=M_{13}=M_{23}=0
$$

e inversamente; così pure l'annullarsi della (21) implica

$(\mathrm{n})_{1}$

$$
N_{12}=N_{13}=N_{23}=0
$$

e inversamente. Di più si ha identicamente

$(\mathrm{mn})$

$$
M_{12}=N_{12}=H
$$

e inoltre le (m), hanno sempre come necessaria conseguenza le $(\mathrm{n})_{1}$.

Ciò premesso osserviamo che la caratteristica di

$$
M_{12}=N_{12}=H=\mid \begin{array}{ll}
{\left[u\left(a u^{\prime}+b u\right)\right]_{x_{1}}^{x_{3}}} & {\left[u\left(a v^{\prime}+b v\right)\right]_{x_{1}}^{x_{2}}} \\
{\left[v\left(a u^{\prime}+b u\right)\right.} & ]_{x_{1}}^{x_{2}}\left[v\left(a v^{\prime}+b v\right)\right]\right]_{x_{1}}^{x_{x_{1}}}
\end{array}
$$

non può essere zero, che altrimenti dovrebbe aversi simultaneamente

$$
a_{1} u_{1}{ }^{\prime}+b_{1} u_{1}=0, \quad a_{1} v_{1}{ }^{\prime}+b_{1} v_{1}=0,
$$

e quindi $a_{1}=b_{1}=0$, ciò che è assurdo.

Supponiamo allora che uno degli elementi di $H$, il primo ad esempio, sia diverso da zero.

In questo supposto le $(\mathrm{m})_{1}$ e le $(\mathrm{n})_{1}$ risultano soddisfatte allora e solo quando si abbia rispettivamente

$(\mathrm{m})_{2}$

$$
\begin{aligned}
& M_{12}=M_{13}=0 \\
& N_{12}=N_{13}=0 .
\end{aligned}
$$

E poichè le $(\mathrm{n})_{1}$ sono oonseguenza delle $(\mathrm{m})_{1}$, le $(\mathrm{n})_{2}$ lo saranno delle $(\mathrm{m})_{2}$. Ne consegue, a causa della (mn), che quando $H=0$, la condizione

$(\mathbf{m})^{\prime}$

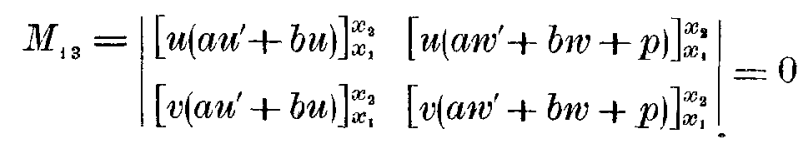


ha come conseguenza necessaria l'altra

$(n)^{\prime}$

$$
N_{13}=\left|\begin{array}{ll}
\left\lfloor u\left(a u^{\prime}+b u\right)\right]_{x_{1}}^{x_{3}} & {\left[p u-\int_{x_{1}}^{x}\left(p^{\prime}-q\right) u d x\right]_{x_{1}}^{x_{2}}} \\
{\left[v\left(a u u^{\prime}+b u\right)\right]_{x_{1}}^{x_{2}}} & {\left[p v-\left.\int_{x_{1}}^{x}\left(p^{\prime}-q\right) v d x\right|_{x_{1}} ^{x_{2}}\right.}
\end{array}\right| .
$$

Ed ora si osservi che tanto la $(\mathrm{m})^{\prime}$ quanto la $(\mathrm{n})^{\prime}$ sono lineari nei valori $p_{1}$ e $p_{2}$ - di $p(x)$ agli estremi $x_{1}$ e $x_{z}$ - dai quali valori si possono considerare indipendenti $u, v, w$, quindi i coefficienti di $p_{1}$ e $p_{2}$ e il ter. mine noto nelle $(\mathrm{m})^{\prime}$ ed $(\mathrm{n})^{\prime}$.

E poichè comunque con due valori $p_{1}$ e $p_{2}$ si soddisfi alla $(\mathrm{m})^{\prime}$ ne risulta sempre soddisfatta la $(\mathrm{n})^{\prime}$, concluderemo che le (m)' ed (n)' sono equivalenti, e che quindi la condizione $(26)_{1}$ è equivalente alla condizione (21) c. v. d.

\section{Un teorema sugli autovalori.}

Dal precedente teorema VI e dal primo teorema del par. I deduciamo altresi quest'altro sui valori eccezionali del parametro $\rho$.

Teorema VII. - Condizione necessaria e sufficiente perchè l'intervallo $(0,1)$ sia sfornito di valori (eccezionali) di p pei quali è possibile soddisfare al sistema

$$
\left\{\begin{array}{l}
\left(a z^{\prime}\right)+\rho\left(b^{\prime}-c\right) z=0 \\
{\left[z\left(a z^{\prime}+\rho b z\right]_{x_{1}}^{x_{2}} \leq 0,\right.}
\end{array}\right.
$$

con soluzione diverse da quella nulla identicamente, è che $\left(\mathrm{x}_{1}, \mathrm{x}_{2}\right)$ sia privo di punti coniugati rispelto alla equasione

$$
\left(a z^{\prime}\right)^{\prime}+\left(b^{\prime}-c\right) z=0,
$$

e sia inoltre soddisfatta la condizione (26) o la (26) $)_{1}$.

In particolare quindi: se queste condizioni sono soddisfatte, il pì̀ piccolo valore eccezionale positivo del $\rho$ del sistema

$$
\begin{gathered}
\left(a z^{\prime}\right)^{\prime}+\rho\left(b^{\prime}-c\right) z=0 \\
\left(a z^{\prime}+\rho b z\right)_{x_{2}}=\left(a z^{\prime}+\rho b z\right)_{x_{1}}=0
\end{gathered}
$$

è maggiore e uguale ad uno.

Nota. - Questo teorema è suscettibile di una notevole estensione al caso che l'intervallo nel quale si vuole assicurare o escludere l'esistenza di valori 
eccezionali di $\rho$ relativi al sistema (27) sia qualsiasi, e il coefficiente di $z$ nella $(4)_{p}$ dipenda comunque da $\rho$ (\%) Preciseremo la cosa in un prossimo lavoro.

UN' APPLICAZIONE. - Prendiamo a considerare il caso particolare (menzionato nella introduzione) in cui l'equazione delle estremali si riduce alla forma semplice seguente

$$
\left(a y^{\prime}\right)^{\prime}=q-p^{\prime}
$$

e quindi quella di JaOOBI a quest'altra

nel quale cioè

$$
\left(a z^{\prime}\right\}^{\prime}=0
$$

$$
b^{\prime}-c=0 \text { in }\left(x_{1}, x_{2}\right) .
$$

Come integrali particolari dell'equazione (28) prenderemo i seguenti

$$
u=1, \quad v=a_{1} \int_{x_{1}}^{\infty} \frac{d x}{a} .
$$

Notiamo subito intanto ohe la condizione a) di cui ai precedenti teoremi V e VI è sempre soddisfatta.

Circa la condizione b) si ha poi

$$
H=\left|\begin{array}{rr}
1 & a_{1} \int_{x_{1}}^{x_{3}} \frac{d x}{a} \\
-1 & 0
\end{array}\right|\left|\begin{array}{lc}
b_{2} & a_{1}+a_{1} b_{2} \int_{x_{1}}^{x_{8}} \frac{d x}{a} \\
b_{1} & a_{1}
\end{array}\right|=a_{1}^{2} \int_{a_{1}}^{x_{3}} \frac{d x}{a}\left\{b_{2}-b_{1}-b_{1} b_{2} \int_{x_{1}}^{x_{2}} \frac{d x}{a}\right\},
$$

mentre la forma (21) diventa

$$
\left(b_{2}-b_{4}\right) g_{1}^{2}+2\left(a_{1} b_{2} \int_{x_{1}}^{x_{2}} \frac{d x}{a}\right) g_{1} g_{2}+\left(a_{1}^{2} \int_{x_{1}}^{x_{2}} \frac{d x}{a}\right)\left(1+b_{2} \int_{x_{1}}^{x_{2}} \frac{d x}{a}\right) g_{2}^{2} \text {. }
$$

Ne consegue che se

$$
H>0, \quad \text { e } \quad b_{2}-b_{1}>0,
$$

da cui si trae:

$$
1+b_{2} \int_{x_{1}}^{x_{2}} \frac{d x}{a}>0, \quad 1-b_{1} \int_{x_{1}}^{x_{2}} \frac{d x}{a}>0
$$

(i) Cfr. G. Mammana, l. e., cap. IIT. 
il funzionale

$$
J[y]=\int_{x_{1}}^{x_{2}}\left(a y^{\prime 2}+2 b y y^{\prime}+b^{\prime} y^{2}+p y^{\prime}+q y\right) d x
$$

è fornito di minimo assoluto in $(G)$, tale minimo inoltre è proprio ed è realizzato dall' unica estremale soddisfacente alle condizioni di trasversalita.

Se invece $H=0$, una almeno delle due disuguaglianze

$$
b_{2}-b_{2} \geq 0, \quad 1+b_{2} \int_{x_{1}}^{x_{2}} \frac{d x}{a} \geq 0
$$

è realizzata in senso forte, ed inoltre

$$
\left\|b_{2} ; \quad 1+b_{2} \int_{x_{1}}^{x_{2}} \frac{d x}{a} ; \int_{x_{1}}^{x_{3}}\left(q-p^{\prime}\right) d x+b_{2} \int_{x_{1}}^{x_{2}} \frac{d x}{a} \int_{x_{1}}^{x_{2}} \frac{d x}{a}\left(q-p^{\prime}\right) d x+p_{2}\right\|^{2}=0
$$

allora esistono infinite (una semplice infinita) estremali soddisfacenti alle condizioni di trasversalità, ciascuna delle quali realizza il minimo assoluto di $J[y]$ in $(G)$. 